\title{
Ritmos circadianos de consumo alimentar nos lanches e refeições de adultos: aplicação do semanário alimentar
}

\section{Food consumption circadian rhythms in adult snacks \\ and meals: application to weekly menu}

Heide GAUCHE

Maria Cristina Marino CALVO²

Maria Alice Altenburg DE ASSIS

\section{R E S U M O}

\section{Objetivo}

Este estudo descreve o perfil alimentar de indivíduos saudáveis, em seu ambiente natural, a partir da análise da ingestão de energia e macronutrientes nos lanches e refeições e sua distribuição circadiana.

\section{Métodos}

Dezessete voluntários, professores e funcionários técnico-administrativos de uma instituição de ensino de Florianópolis com idade média de $46,71( \pm 2,2)$ anos, e índice de massa corporal médio de $24,93( \pm 0,9) \mathrm{kg} / \mathrm{m}^{2}$ registraram, durante sete dias consecutivos, o tipo e a quantidade de alimentos e bebidas consumidos, especificando o modo de preparo, o tipo de evento alimentar (refeição ou lanche), a hora do dia e o dia da semana.

\section{Resultados}

Realizaram-se, em média, 2,7 refeições e 3,2 lanches diariamente. Os lanches e as refeições mostraram diferenças entre si, tanto em relação à proporção de macronutrientes quanto ao valor energético total. As refeições forneceram cerca de três vezes mais calorias que os lanches e foram compostas, predominantemente, por proteínas e lipídios, enquanto nos lanches predominaram os carboidratos. A hora do dia também mostrou exercer influência no consumo.

\section{Conclusão}

No período das $12 \mathrm{~h}$ às $15 \mathrm{~h} 59 \mathrm{~min}$, o consumo foi significativamente maior que nos demais momentos do dia, mas não se observou um aumento significativo do consumo energético total nos dias do fim de semana.

Termos de indexação: conduta na alimentação; estado nutricional; ritmos circadianos; semanário alimentar.

\footnotetext{
1 Iniciação Científica PIBIC/CNPq, Departamento de Nutrição, Centro de Ciências da Saúde, Universidade Federal de Santa Catarina.

2 Departamento de Saúde Pública, Centro de Ciências da Saúde, Universidade Federal de Santa Catarina. Florianópolis, SC, Brasil.

3 Departamento de Nutrição, Centro de Ciências da Saúde, Universidade Federal de Santa Catarina. Caixa Postal 476, Trindade, 88040-900, Florianópolis, SC, Brasil. Correspondência para/Correspondence to: M.A.A. DE ASSIS.
} 


\section{A B S T R A C T}

\section{Objective}

This study describes the food profile of healthy individuals in their natural environment, analyzing the energy and macronutrient ingestion during meals and snacks and their circadian distribution.

\section{Methods}

Seventeen volunteers, professors and administrative technicians of an educational institution in Florianópolis, Brazil, with an average age of $46.71( \pm 2.2)$ years, and average body mass index of $24.93( \pm 0.9) \mathrm{kg} / \mathrm{m}^{2}$, registered the type and quantity of food and drink consumed during seven consecutive days, specifying the type of preparation, the eating event (meal or snack), the hour of the day and the day of the week.

\section{Results}

On average, they consumed 2.7 meals and 3.2 snacks every day. The snacks differed from the meals in both the size and proportion of the macronutrients and in the total energy value. The meals provided about three times more calories than the snacks and were mostly composed of proteins and lipids, whilst the snacks were mostly carbohydrate. The time of day also exerted an influence on consumption.

\section{Conclusion}

In the period from 12 hours to 15 hours 59min, the consumption was significantly greater than during the rest of the day. There was no difference in total caloric consumption between weekends and weekdays.

Indexing terms: feeding behavior; nutritional status; circadian rhythms; weekly food diary.

\section{N T R O D U Ç Ã O}

O estudo do comportamento alimentar é complexo e detalhado. A forma tradicional de estudá-lo não considera diversos fatores que causam variações no consumo alimentar, e que estão relacionados ao contexto social do indivíduo, seu estado emocional e fisiológico ${ }^{1}$. Por esse motivo, este estudo fez a avaliação do comportamento alimentar de indivíduos em seu ambiente natural, a fim de não eliminar a influência dessas variáveis. Para isso, foi utilizado um instrumento denominado semanário alimentar, que possibilita a pesquisa observacional sem a influência do pesquisador ou do observador ${ }^{2}$.

Alguns estudos já publicados identificaram variáveis que exercem influência no consumo alimentar. Marshall \& Bell ${ }^{3}$ observaram que pessoas de diferentes culturas, ao respeitar hábitos e costumes, combinam os alimentos que farão parte de suas refeições de maneiras distintas. Sexo, idade e até fatores genéticos podem exercer influência no consumo alimentar, alterando o valor energético consumido, a freqüência e o horário de realização das refeições, a quantidade e a proporção de macronutrientes ${ }^{4-6}$. O próprio local de realização da refeição ou do lanche pode definir o consumo, por limitar ou ampliar a escolha de alimentos ${ }^{3}$.

O número de eventos alimentares também pode variar, dependendo da quantidade de alimentos, bem como do nível de estresse a que o indivíduo está submetido ${ }^{7}$. Recentemente, o estudo dos ritmos circadianos do consumo alimentar e seus efeitos no metabolismo tem merecido atenção da comunidade científica ${ }^{8-10}$.

O consumo alimentar dos seres humanos é composto por eventos alimentares - podendo ser denominados de refeições e lanches - que variam em número e composição. Os lanches podem fornecer um valor significativo de energia e nutrientes, cuja natureza e qualidade podem variar de acordo com o contexto sociocultural, o peso corporal, a idade e o sexo ${ }^{8}$.

As pessoas caracterizam os eventos alimentares de maneiras distintas, geralmente identificando-os como café da manhã, almoço e jantar - além de lanches -, considerando a hora do dia em que são realizados e os tipos de alimentos consumidos ${ }^{11}$, resultando em aportes energéticos e de macronutrientes diferentes entre os períodos da manhã, tarde e noite ${ }^{8}$. 
Neste estudo, avaliou-se o perfil nutricional de eventos alimentares num grupo de funcionários públicos da cidade de Florianópolis que registraram o consumo de alimentos e bebidas durante sete dias consecutivos. O objetivo foi comparar a freqüência, o teor energético e de macronutrientes de lanches e refeições, entre os dias de semana e de fins de semana, bem como os ritmos circadianos de consumo energético.

\section{M É T O D O S}

O Colégio de Aplicação da Universidade Federal de Santa Catarina (UFSC) é uma escola de primeiro e segundo graus, localizada no campus universitário, em Florianópolis. Na época da pesquisa, seu quadro funcional era constituído por 85 professores efetivos, 21 professores substitutos e 30 funcionários técnico-administrativos. Dos 136 profissionais que foram convidados a participar da pesquisa e esclarecidos sobre seu objetivo, apenas $12,5 \%$ (15 mulheres e 2 homens) registraram todos os dados solicitados durante os 7 dias consecutivos. Três funcionários começaram a preenchê-lo, porém não o completaram, devido à dedicação exigida para o correto preenchimento.

Os voluntários deveriam registrar, por escrito, durante sete dias consecutivos, os tipos e as quantidades de alimentos e bebidas consumidos, especificando o modo de preparo, os horários dos eventos alimentares e outras informações de sensação e percepção. Os dados foram coletados no mês de outubro de 2003. O estudo foi aprovado pelo Comitê de Ética em Pesquisa com Seres Humanos da UFSC.

O método do semanário alimentar (weekly food diary) foi utilizado a partir do começo dos anos da década de 80, e vários trabalhos foram publicados em revistas científicas de nível internacional. A versão brasileira foi denominada "semanário alimentar"12, e consiste no registro de cada alimento e bebida consumidos durante sete dias consecutivos, além da quantidade e do modo de preparo do alimento, hora de ingestão e tipo de evento alimentar - refeição ou lanche. São também registradas informações diárias sobre os horários de dormir e acordar, e a qualidade do sono. Percepções e sensações quanto à atividade física, à intensidade da fome e da sede, à aparência da refeição, ao estado de espírito (contente, deprimido, calmo, ansioso) também foram registradas antes e após cada evento alimentar.

O semanário alimentar ${ }^{12}$ é um instrumento no formato de um bloco, composto por diversas folhas. Cada evento alimentar, seja uma refeição ou um lanche, deve ser registrado em uma folha única. Todos os detalhes com relação à atividade física, sensações de sede, fome, calma/ansiedade, depressão/alegria, bem como informações com relação ao próprio alimento - se parece estar apetitoso ou não - deveriam ser percebidos pelo voluntário e registrados na folha correspondente. Portanto, não se trata apenas do registro do consumo alimentar; representa, antes, a descrição do ambiente como um todo, por meio do registro do local, horário, dia da semana, tipo de evento alimentar e número de pessoas presentes. Cada folha contém 21 campos a serem preenchidos. $O$ voluntário utiliza, em média, 6 folhas por dia, totalizando 126 informações registradas em apenas um dia da semana. Sete dias exigem, portanto, a atenção no preenchimento de, em média, 882 dados referentes apenas ao comportamento alimentar. Há também dez campos que devem ser preenchidos com informações referentes à qualidade do sono diário. Esses números refletem a riqueza das informações que devem ser preenchidas pelos voluntários.

Em um primeiro encontro, os sujeitos foram orientados a registrar as informações do consumo alimentar de apenas um dia, da forma mais detalhada possível. Em seguida, foram contatados para revisar as informações registradas, corrigir os problemas e solucionar as possíveis dúvidas. Os dados desse primeiro teste não foram utilizados para a análise. Somente após esse dia, o participante recebeu o bloco para registro semanal. 
Com o intuito de reforçar a motivação dos avaliados e de aumentar a confiança e a habilidade dos participantes no preenchimento do semanário, o pesquisador manteve contato diário com os voluntários, em hora e local pré-estabelecidos, evitando-se, assim, ambigüidades de informações e falta de dados.

\section{Avaliação do estado nutricional}

O índice de massa corporal (IMC) $\left(\mathrm{kg} / \mathrm{m}^{2}\right)$, obtido a partir da relação peso corporal e altura, foi utilizado para a classificação do estado nutricional. Foram utilizados os pontos de corte do IMC propostos pela World Health Organization $(\mathrm{WHO})^{13}$ para efetuar o diagnóstico do estado nutricional.

O peso corporal $(\mathrm{kg})$ e a altura $(\mathrm{cm})$ foram obtidos no início do estudo, no momento das explicações quanto ao preenchimento do semanário alimentar e da entrega do formulário de um dia. O peso foi obtido utilizando-se uma balança eletrônica digital (Marte), com precisão de $100 \mathrm{~g}$, estando os participantes com roupas leves e sem sapatos. A altura foi aferida com fita métrica fixada na parede sem rodapés.

Os alimentos registrados no semanário alimentar de todos os participantes da pesquisa foram repassados para o software Diet Pro ${ }^{14}$. As informações sobre a composição nutricional de alimentos e bebidas, não presentes no banco de dados desse software, foram obtidas a partir da tabela de composição da Universidade de São Paulo ${ }^{15}$, Repertório Geral dos Alimentos ${ }^{16}$, Tabela de Composição Química dos Alimentos ${ }^{17}$, por meio de rótulos comerciais ou pela elaboração de fichas de preparação, seguindo-se o método proposto por McCance \& Widdowson ${ }^{18}$.

Os valores absolutos e percentuais de calorias totais e dos macronutrientes dos itens alimentares que compunham cada evento alimentar foram repassados para um programa desenvolvido em Epi Info 6.0419, por intermédio do qual foram efetuados o gerenciamento e a análise estatística dos dados. Cada evento alimentar foi identificado como sendo uma refeição ou um lanche, com base na definição do próprio indivíduo. As quantidades de energia provenientes de carboidratos, proteínas, gorduras e álcool de cada evento alimentar foram somadas, tendo-se, depois, calculado a média diária individual para os dias de semana (de segunda a sexta-feira) e dias de fim de semana (sábado e domingo). A partir das médias individuais diárias, estabeleceu-se a média do grupo. Para analisar a distribuição circadiana de consumo energético, dividiu-se o dia em cinco períodos (1h - 4h59min; 5h - 11 h59min; 12h-15h59min; 16h-19h59min; 20h-00h59min), procedendo-se à análise da variação da ingestão energética entre esses períodos ${ }^{9}$.

Os dados foram apresentados em valores médios e erro padrão da média (EPM). Diferenças entre os tipos de eventos alimentares - realizados durante a semana e no fim de semana e nos cinco períodos do dia - foram testadas por meio de análise de variância (ANOVA). Adotou-se o nível de significância de 0,05.

\section{RES U LT A D OS}

A complexidade inerente ao instrumento de coleta de dados limitou a adesão à pesquisa, conformando um grupo de 17 voluntários, independentemente do sexo, com idade média $( \pm$ EPM) de $46,7( \pm 2,2)$ anos.

O valor médio do IMC esteve no limite máximo da faixa de normalidade $\left(24,9 \pm 0,9 \mathrm{~kg} / \mathrm{m}^{2}\right)$, segundo a $\mathrm{WHO}^{13}$. Quatro mulheres apresentaram sobrepeso (IMC entre 25 - 29,9) e três mulheres apresentaram obesidade (IMC entre 30 - 34,9). Nenhum participante apresentou baixo peso.

A ingestão energética diária média ( \pm EPM) do grupo foi de $2.144,1( \pm 105,4) \mathrm{kcal}$, assim distribuídas: $48,7 \%( \pm 1,1)$ provenientes de carboidratos, 33,9\% $( \pm 1,1)$ de lipídios, $15,1 \%$ $( \pm 0,5)$ de proteínas e 2,3\% $( \pm 0,6)$ de álcool.

Foram realizados 692 eventos alimentares (377 lanches e 315 refeições). Cerca de 24,0\% 
do valor energético ingerido diariamente foram fornecidos pelos lanches e cerca de 76,0\% foram provenientes das refeições.

São apresentadas as freqüências diárias de lanches e refeições e o consumo médio por evento alimentar de energia e de macronutrientes (média \pm erro padrão da média) (Tabela 1 ). Registraram-se 5,9 $( \pm 0,3)$ eventos alimentares por dia, obtendo-se, em média, 3,2 lanches e 2,7 refeições diárias. As refeições apresentaram valor energético significativamente maior que os lanches e, em termos absolutos, maior quantidade dos macronutrientes.

Tabela 1. Consumo médio por evento alimentar de energia e de macronutrientes (média \pm desvio-padrão) nos lanches e refeições de adultos de Florianópolis, 2003.

\begin{tabular}{|c|c|c|c|c|c|c|}
\hline & \multicolumn{2}{|c|}{ Lanches } & \multicolumn{2}{|c|}{ Refeições } & & \multirow{2}{*}{$F$} \\
\hline & $\bar{x}$ & $\mathrm{DP}$ & $\bar{x}$ & DP & & \\
\hline Média de eventos & $3,2 \pm$ & 0,3 & $2,7 \pm$ & 0,4 & 0,10 & 2,4 \\
\hline \multicolumn{7}{|c|}{ Consumo por evento (kcal) } \\
\hline Energia & $191,8 \pm$ & 21,7 & $586,5 \pm$ & 33,3 & 0,00 & 98,8 \\
\hline Carboidratos & $113,3 \pm$ & 11,8 & $265,7 \pm$ & 14,7 & 0,00 & 65,7 \\
\hline Lipídios & $54,0 \pm$ & 7,5 & $210,9 \pm$ & 17,0 & 0,00 & 71,6 \\
\hline Proteínas & $21,8 \pm$ & 3,9 & $96,9 \pm$ & 6,3 & 0,001 & 102,8 \\
\hline Álcool & $2,7 \pm$ & 1,1 & $13,0 \pm$ & 3,9 & 0,02 & 6,4 \\
\hline
\end{tabular}

Os lanches foram proporcionalmente mais ricos em carboidratos ( $p=0,000 F=30,6)$, enquanto as refeições contribuíram, proporcionalmente, com uma quantidade significativamente maior de proteínas $(p=0,000 F=49,6)$ e lipídios $(p=0,001$ $F=14,9)$. O consumo proporcional de álcool não mostrou diferença significativa entre refeições e lanches $(p=0,6 F=0,3)$ (Figura 1).

No período das $12 \mathrm{~h}$ às $15 \mathrm{~h} 59 \mathrm{~min}$, o consumo energético mostrou-se significativamente maior do que em outros períodos ( $p=0,000 F=33,64)$. 0 período de menor consumo alimentar foi registrado no horário da madrugada, compreendido entre $1 \mathrm{~h}$ e $4 \mathrm{~h}: 59 \mathrm{~min}$ (Figura 2).

Não houve diferença estatisticamente significativa entre a ingestão energética diária média registrada nos dias de semana - segunda a sexta-feira - $(2099,9 \pm 106,6 \mathrm{kcal})$ e nos dias de fim de semana - sábado e domingo - (2256,6 \pm $154,1 \mathrm{kcal})(p=0,409 F=0,69)$. Tanto em uns como em outros, o pico do consumo energético foi registrado no período das $12 \mathrm{~h}$ às $15 \mathrm{~h} 59 \mathrm{~min}$. No fim de semana, esse pico foi significativamente superior, tanto aos demais períodos, quanto ao mesmo período dos dias de semana $(p<0,05)$. Entre os demais períodos, quando analisados intervalos iguais do dia, não foram observadas diferenças estatisticamente significativas entre a ingestão energética nos dias de semana e nos dias do fim de semana (Figura 3).

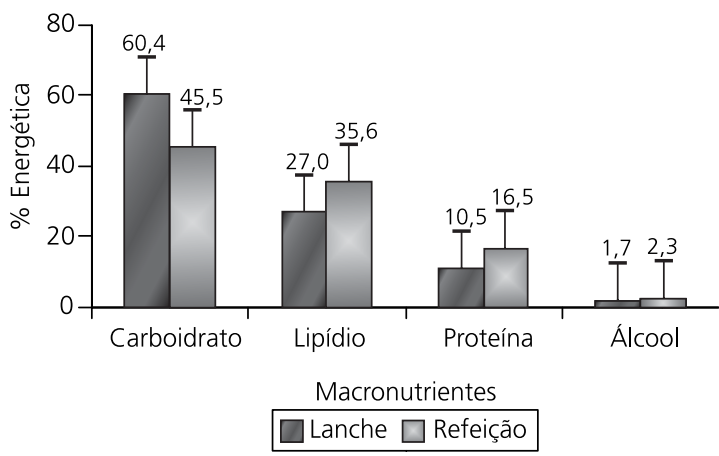

Figura 1. Composição nutricional de lanches e refeições, expressa como percentagem do total de energia proveniente de carboidratos, lipídios, proteínas e álcool. Florianópolis, 2003

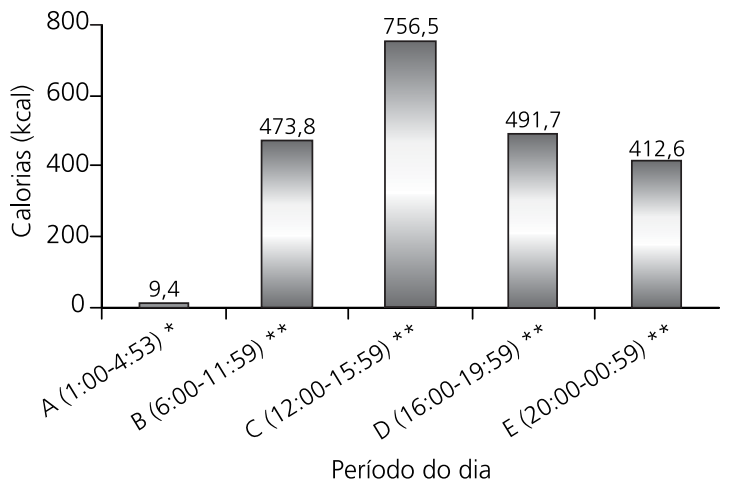

Figura 2. Distribuição circadiana do consumo energético (média e intervalo de confiança) em cinco períodos. Florianópolis, 2003

Nota: *Média menor que todos os outros períodos $(p<0,05)$,

**Média de $C$ maior que $B, D$ e $E(p<0,05)$. 


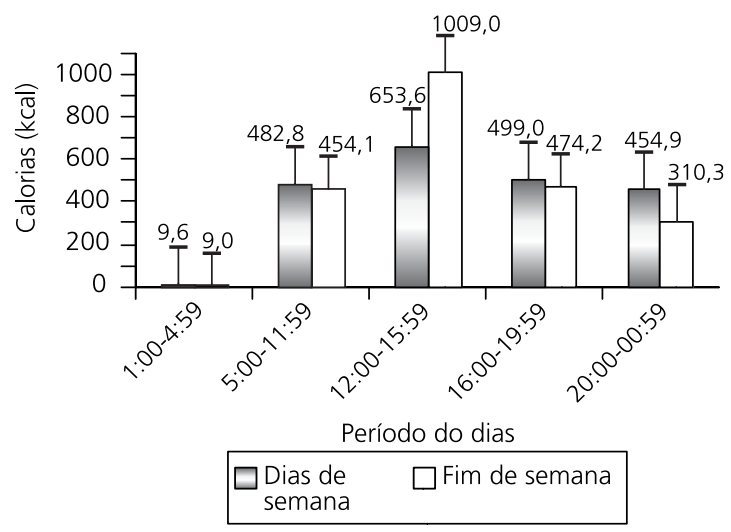

Figura 3. Distribuição circadiana do consumo energético (média e intervalo de confiança) em cinco períodos dos dias de semana e dias de fins de semana, expresso como média diária do consumo energético (kcal). Florianópolis, 2003

\section{I S C U S S Ã O}

Neste trabalho estudou-se o perfil nutricional de lanches e refeições em termos de contribuição energética e de macronutrientes, bem como a distribuição circadiana de ingestão energética durante a semana e o fim de semana, numa amostra de funcionários públicos de Florianópolis. Entre as limitações deste estudo, cita-se o reduzido número de participantes da pesquisa (12,5\% dos funcionários da instituição). Evidencia-se, entretanto, que apesar do pequeno número de participantes, os dados obtidos referem-se ao registro de 17 indivíduos em 7 dias consecutivos, analisando-se 119 dias de consumo e 692 eventos alimentares (lanches e refeições).

Poucos estudos publicados, especialmente no Brasil, têm obtido dados de consumo energético e de macronutrientes relativos a sete dias consecutivos, porque o instrumento de coleta demanda tempo, dedicação e precisão, o que, certamente, contribuiu para a baixa adesão dos convidados.Há estudos internacionais que utilizaram o semanário alimentar com número de participantes significativamente maior; todavia isso parece ter sido possível pelo fato de os participantes terem sido remunerados ${ }^{1}$.

Percebe-se que, apesar do grande número de pesquisas já realizadas com relação ao consumo alimentar de diferentes populações, pouco se tem publicado a respeito do comportamento alimentar em si, como a periodicidade do consumo alimentar, a freqüência, a composição nutricional dos eventos alimentares e a distribuição energética no decorrer das 24 horas de um dia ${ }^{20}$. Este trabalho, portanto, constitui contribuição importante para o estudo desses determinantes do comportamento alimentar.

As características antropométricas da população avaliada mostraram que, em média, o estado nutricional dos participantes encontra-se no limite da faixa de normalidade. Segundo Ell et al. ${ }^{21}$, que estudaram o perfil antropométrico de 647 funcionários do Banco do Brasil, no Rio de Janeiro, a proporção de indivíduos com excesso de peso aumentou com a idade, tanto para homens quanto para mulheres. Para as mulheres, a proporção de obesas com idade acima de 40 anos foi duas vezes maior do que nas mulheres com idade abaixo de 34 anos. A média da idade obtida neste estudo pode ser, portanto, uma variável com significativa influência no IMC.

A definição de refeição e de lanche é um tema que vem merecendo atenção na literatura científica. Em alguns estudos, definições quantitativas são usadas para diferenciar refeições de lanches, baseadas em critérios objetivos; por exemplo, uma refeição deve conter pelo menos uma quantidade específica de energia ${ }^{9,10,22,23}$, ou conter certa quantia de diferentes tipos de alimentos ${ }^{24}$. Neste estudo, da mesma forma que em Bellisle et al. ${ }^{8}$, a identificação do evento alimentar como sendo uma refeição ou lanche ficou a critério do próprio participante. A freqüência diária de eventos alimentares realizados pela amostra de adultos de Florianópolis $(5,9)$ foi maior do que a relatada para adultos franceses ${ }^{8}$, numa pesquisa que utilizou o mesmo instrumento de coleta de dados: o semanário alimentar. Enquanto o número de refeições realizadas por adultos franceses e de Florianópolis foi o mesmo $(2,7$ versus 2,7$)$ no que se refere a lanches, a amostra de adultos de Florianópolis apresentou um número maior do que a dos franceses $(3,2$ versus 1,3$)$. 0 hábito alimentar francês de realizar três refeições 
diárias vem se modificando nos últimos anos, podendo ser conseqüência de um novo estilo de vida ${ }^{25}$.

Os achados deste estudo também apontam para uma diminuição da freqüência de três refeições principais, característica dos hábitos brasileiros, e merecem atenção em estudos sobre o consumo alimentar no Brasil, uma vez que uma ou mais refeições principais podem estar sendo substituídas por lanches. A importância nutricional do consumo de lanches entre as principais refeições encontra nos estudos publicados diferentes visões. Enquanto alguns estudos mostraram que esse hábito pode facilitar o aumento do consumo para além das necessidades energéticas, fornecendo quantidades importantes de nutrientes de baixa densidade nutricional, outros relataram que os lanches contribuíram significativamente para aumentar a qualidade nutricional da dieta ${ }^{20}$.

Alguns estudos mostraram uma associação inversa entre a freqüência de consumo e o peso corporal $^{26}$ e outros encontraram associações positivas entre a freqüência diária de eventos alimentares e parâmetros fisiológicos, tais como redução do colesterol sangüíneo e melhora de tolerância à glicose $\mathrm{e}^{27,28}$.

Nesta pesquisa, a composição dos lanches apresentou-se diferente daquela das refeições, diferindo entre si no conteúdo energético - as refeições somaram, em média, o triplo de calorias - e no conteúdo proporcional de nutrientes - os lanches apresentaram mais carboidratos e menos proteína e lipídios. Estudos realizados na França ${ }^{8}$ e na Finlândia ${ }^{29}$ também mostraram que os lanches contêm proporcionalmente mais carboidratos e menos proteínas e lipídios do que as refeições, corroborando os dados deste estudo. A contribuição energética dos lanches para o consumo energético total diário do grupo de adultos de Florianópolis (24,0\%) foi maior do que o relatado em estudos realizados na França ${ }^{8}$ $(18,5 \%)$ e na Inglaterra ${ }^{30}$, com mulheres de meia idade $(21,4 \%)$. As diferenças encontradas entre o nosso estudo e os realizados em diferentes países sugerem que o contexto sociocultural exerce importante influência sobre o perfil nutricional das refeições e dos lanches.

Quando se comparou o consumo energético médio dos dias do fim de semana com o dos dias de semana, foi encontrado um acréscimo de $7,5 \%$ de calorias por dia $(156,7 \mathrm{kcal})$ no fim de semana, mas as diferenças não foram estatisticamente significativas. Esses resultados foram similares aos de um estudo realizado na Austrália ${ }^{31}$, que relatou um acréscimo de $152 \mathrm{kcal}$ para o fim de semana, e aos registrados por Castro $^{23}$ na população americana, com um acréscimo de $145 \mathrm{kcal}(8,0 \%)$. Castro ${ }^{23}$ não observou diferenças significativas do consumo energético entre os dias de semana (segunda-feira a quinta-feira), porém observou aumento significativo do consumo energético e de macronutrientes no fim de semana (sexta-feira a domingo), associado à maior duração de tempo das refeições nesse período.

Em outro estudo, realizado nos Estados Unidos $^{32}$, com uma amostra representativa da população (1994-1996), foi relatado um consumo estatisticamente diferente entre os dias de semana (de segunda a quinta-feira) e os dias de fim de semana (sexta-feira a domingo); na faixa etária compreendida entre 19 e 50 anos, o aumento de calorias ingeridas nos fins de semana em relação aos dias de semana foi de $115 \mathrm{kcal} / \mathrm{dia}$.

Os resultados deste estudo mostraram um consumo energético uniforme durante a semana, com pequenas variações nos períodos do dia. Essas variações parecem ser conseqüência da característica específica das refeições realizadas nos diferentes períodos do dia, que são compostas, habitualmente, por tipos e quantidades similares de alimentos. Observou-se que o pico de consumo energético ocorreu em dois momentos distintos: em torno do meio-dia (12h - 15h59min) e no início da noite (16h - 19h59min), períodos em que, tradicionalmente, são realizados o almoço e o jantar. Uma análise semelhante a essa foi realizada por Bellisle \& Dalix ${ }^{22}$, que revelou um consumo energético semelhante entre o almoço e o jantar. 
Os ritmos circadianos e diurnos afetam a ingestão de alimentos; outros estudos têm sugerido que, com o passar do dia, o tamanho da refeição aumenta, enquanto os intervalos após as refeições e as taxas de saciedade decrescem $^{8,10}$. Num estudo com uma população de 375 homens e 492 mulheres americanas ${ }^{10}$, em que foi aplicado o método do semanário alimentar, a proporção de ingestão observada pela manhã foi negativamente correlacionada com a ingestão total, enquanto a proporção ingerida em períodos mais tardios do dia foi positivamente correlacionada com a ingestão total. O autor sugeriu que a ingestão durante a manhã pode apresentar maior poder de saciedade e reduzir a quantidade total ingerida durante o dia, e que a ingestão no período da noite tem menor efeito sobre a saciedade, resultando em aumento da ingestão total diária.

Num estudo para caracterizar os padrões de refeições em relação à obesidade de mulheres suecas $^{33}$, foi observado que a freqüência das refeições das mulheres obesas $(6,1 / \mathrm{dia})$ foi significativamente maior do que a das mulheres não obesas (5,2/dia). Também foi relatado que as mulheres obesas consumiam mais refeições no final do dia e à noite.

\section{O N C L U S Ã O}

Este estudo apresenta apenas alguns dos aspectos que podem ser trabalhados com as informações obtidas com o semanário alimentar e que estão relacionadas ao comportamento alimentar e seus determinantes.

Entre os aspectos positivos do perfil alimentar dos voluntários, destacam-se um consumo energético total adequado para a faixa etária e atividade de trabalho, a proporção adequada de calorias protéicas e o número adequado de refeições diárias, predominando as calorias provenientes das refeições em relação aos lanches. A distribuição energética durante os sete dias da semana não mostrou disparidades, revelando um consumo alimentar equilibrado.
Algumas diferenças no valor energético consumido nos diferentes períodos do dia mostraram a importância das refeições tradicionalmente conhecidas como almoço e jantar, uma vez que foi justamente nos períodos do meio-dia e à noite que foi registrado o maior consumo energético.

A presença dos lanches no dia alimentar dos indivíduos contribuiu para o incremento do consumo energético total diário, às custas, principalmente, de carboidratos e, em menor proporção, de lipídios e proteínas. Tratando-se ou não de um hábito alimentar inadequado, deve-se prestar atenção ao tipo de alimento consumido nesses lanches, para que eles sejam um evento alimentar que não venha trazer prejuízo para a qualidade global da dieta.

Os achados deste estudo, por se referirem a um pequeno grupo de funcionários públicos da cidade de Florianópolis, trazem informações ainda pouco exploradas sobre o perfil nutricional de lanches e refeições da população brasileira. Nesse sentido, esta pesquisa está sendo expandida no intuito de aumentar o número de participantes, estudar a composição em micronutrientes e outros aspectos comportamentais que interferem na freqüência e na composição nutricional de lanches e refeições, bem como de avaliar associações entre o IMC e o consumo energético nos diferentes períodos do dia.

\section{REFERÊ NCIAS}

1. Castro JM. Methodology, correlational analysis, and interpretation of diet diary records of food and fluid intake of free-living humans. Appetite. 1994; 23(2):179-92.

2. Castro JM, Kreitzman SN. A microregulatory analysis of spontaneous human feeding patterns. Physiol Behav. 1985; 35(3):329-35.

3. Marshall D, Bell R. Meal construction: exploring the relationship between eating occasion and location. Food Quality Prefer. 2003; 14(1):53-64.

4. Soriano JM, Moltó JC. Dietary intake and food pattern among university students. Nutr Res. 2000; 20(9):1249-58.

5. Ton Nu C, MacLeod P, Barthelemy J. Effects of age and gender on adolescents' food habits and 
preferences. Food Quality Prefer. 1996; 7(3/4): 251-62.

6. Castro JM. Behavioral Genetics of Food Intake Regulation in Free-Living Humans. Nutrition. 1999; 15(7-8):550-4.

7. Marmonier C, Chapelot D, Louis-Sylvestre J. Effects of macronutrient content and energy density of snack consumed in a satiety state on the onset of the next meal. Appetite. 2000; 34(2):161-8.

8. Bellisle F, Dalix AM, Mennen L, Galan P, Hercberg S, Castro JM, et al. Contribution of snacks and meals in the diet of French adults: a diet-diary study. Physiol Behav. 2003; 79(2):183-9.

9. Assis MA, Kupek E, Nahas MV, Bellisle F. Food intake and circadian rhythms in shift workers with a high workload. Appetite. 2003; 40(2):175-83.

10. Castro JM. The time of day of food intake influences overall intake in humans. J Nutr. 2004; 134(1):104-11.

11. Anderson J, Nydahl M, Gustafson K, Sidenvall B, Fjellström C. Meals and snacks among eldery self-managing and disabled women. Appetite. 2003; 41(2):149-60.

12. Sachs A, Assis MAA, Passos MC. Semanário alimentar. In: Fisberg RM, Slater B, Marchioni DML, Martini LA. Inquéritos alimentares: métodos e bases científicas. São Paulo; 2004. p.32-52.

13. World Health Organization. Obesity: preventing and managing the global epidemic. Geneva; 1998. p.9.

14. Software Diet Pro, 2001. Disponível em: http:// www.agromidia.com.br

15. Tabela brasileira de composição de alimentos, 2001. Disponível em: http://fcf.usp.br/tabela

16. Favier JC, Ripert JI, Toque C, Feinberg M. Repertório geral dos alimentos: tabela de composição. 2.ed. São Paulo: Roca; 1999.

17. Franco G. Tabela de composição química dos alimentos. 9. ed. Rio de Janeiro: Atheneu; 1992.

18. McCance RA, Widdowson ED. The composition of foods. 5th ed. Cambridge; Royal Society of Chemistry; 1991.

19. Dean AG, et al. Epi Info [computer program]. Version 6.04: a word processing, database, and statistics program for epidemiology on micro-computers. Atlanta, Georgia: Centers of Disease Control and Prevention; 1994.

20. Gatenby SJ. Eating frequency: methodological and dietary aspects. Br J Nutr. 1997; 77(Suppl 1): S7-S20.
21. Ell E, Camacho LAB, Chor D. Perfil antropométrico de funcionários de banco estatal no Estado do Rio de Janeiro/Brasil: Índice de massa corporal e fatores sócio demográficos. Cad Saúde Pública. 1999; 15(1):113-22.

22. Bellisle F, Dalix AM. Eating patterns in French subjects studied by the "Weekly Food Diary" Method. Appetite. 1999; 32(1):46-52.

23. Castro JM. Weekly rhythms of spontaneous nutrient intake and meal pattern of humans. Physiol Behav. 1991; 50(4):729-38.

24. Lennernãs M, Anderson I. Food-based Classification of Eating Episodes. Appetite. 1999; 32(1):53-65.

25. Poulain JP. The contemporary diet in France: "de-structuration" or from commensalism to "vagabond feeding". Appetite. 2002; 39(1):43-55.

26. Bellisle F, McDevitt R, Prentice A. Meal frequency and energy balance. Br J Nutr. 1997; 77(Suppl 1):S57-S70.

27. Jenkins DJA. Carbohydrate tolerance and food frequency. Br J Nutr. 1997; 77(Suppl 1):S71-S81.

28. Farshchi HR, Taylor MA, MacDonald A. Beneficial metabolic effects of regular meal frequency on dietary thermogenesis, insulin sensitivity, and fasting lipid profiles in healthy obese women. Am J Clin Nutr. 2005; 81(1):16-24.

29. Roos E, Prättälä R. Meal pattern and nutrient intake among adult Finns. Appetite. 1997; 29(1):11-24.

30. Summerbell CD, Moody RC, Shanks J, Stock MJ, Geissler C. Sources of energy from meals versus snacks in 220 people in four age groups. Eur J Clin Nutr. 1995; 49(1):33-41.

31. Lyons PM, Truswell AS, Mira M, Vizard J, Abraham SF. Reduction of food intake in the ovulatory phase of the menstrual cycle. Am J Clin Nutr. 1989; 49(6):1164-68

32. Haines PS, Hama MY, Guilkey DK, Popkin BM. Weekend eating in the United States is linked with greater energy, fat, and alcohol intake. Obes Res. 2003; 11(8):945-9.

33. Berteus Forslund $H$, Lindroos AK, Sjostrom L, Lissner L. Meal patterns and obesity in Swedish women-a simple instrument describing usual meal types, frequency and temporal distribution. Eur J Clin Nutr. 2002; 56(8):740-7.

Recebido em: 10/3/2005

Versão final reapresentada em: 25/7/2005 Aprovado em 15/8/2005 